Research Paper

\title{
Opposite Effects of Bone Marrow-Derived Cells Transplantation in MPTP-rat Model of Parkinson's Disease: A Comparison Study of Mononuclear and Mesenchymal Stem Cells
}

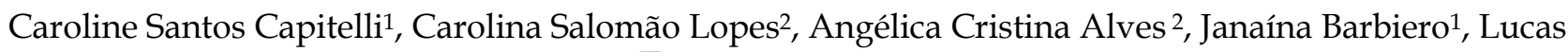
Felipe Oliveira ${ }^{2}$, Valdo José Dias da Silva ${ }^{2 \llbracket *}$, Maria Aparecida Barbato Frazão Vital ${ }^{1^{\star}}$

1. Department of Pharmacology, Paraná Federal University, Curitiba, Paraná, Brazil;

2. Department of Biochemistry, Pharmacology, Physiology and Molecular Biology, Triângulo Mineiro Federal University, Uberaba, Minas Gerais, Brazil.

* Valdo José Dias da Silva and Maria Aparecida Barbato Frazão Vital equally contributed to this study.

$\square$ Corresponding author: Valdo José Dias da Silva, Triângulo Mineiro Federal University, Institute for Biological and Natural Science, Praça Manoel Terra, 330, CEP 38025-015, Uberaba- MG-Brazil. E-mail: valdo@mednet.com.br.

(c) Ivyspring International Publisher. This is an open-access article distributed under the terms of the Creative Commons License (http://creativecommons.org/ licenses/by-nc-nd/3.0/). Reproduction is permitted for personal, noncommercial use, provided that the article is in whole, unmodified, and properly cited.

Received: 2013.12.20; Accepted: 2014.07.17; Published: 20I4.08.03

\begin{abstract}
The I-methyl-4-phenyl-1,2,3,6-tetrahydropyridine (MPTP) animal model is a useful tool to study Parkinson's disease (PD) and was used in the present study to investigate the potential beneficial as well as deleterious effects of systemic bone-marrow mononuclear cell (BMMC) or mesenchymal stem cell (BM-MSC) transplantation. MPTP administration resulted in a breakdown of the blood-brain barrier and motor impairment in the open field test $24 \mathrm{~h}$ after surgery. Three and 7 days after receiving the lesion, the injured animals showed remaining motor impairment compared to the sham groups along with a significant loss of tyrosine hydroxylase-immunoreactive (TH-ir) cells in the substantia nigra pars compacta $(\mathrm{SNPc})$. The MPTP-lesioned rats treated with BMMCs immediately after lesioning exhibited motor impairment similar to the MPTP-saline group, though they presented a significantly higher loss of TH-ir cells in the SNpc compared to the MPTP-saline group. This increased loss of TH-ir cells in the SNpc was not observed when BMMC transplantation was performed $24 \mathrm{~h}$ after MPTP administration. In contrast, in the MPTP animals treated early with systemic BM-MSCs, no loss of TH-ir cells was observed. BMMCs and BM-MSCs previously labeled with CM-Dil cell tracker were found in brain sections of all transplanted animals. In addition, cells expressing CD45, an inflammatory white blood cell marker, were found in all brain sections analyzed and were more abundant in the MPTP-BMMC animals. In these animals, Ibal+ microglial cells showed also marked morphological changes indicating increased microglial activation. These results show that systemic BMMC transplantation did not ameliorate or prevent the lesion induced by MPTP. Instead, BMMC transplantation in MPTP-lesioned rats accelerated dopaminergic neuronal damage and induced motor impairment and immobility behavior. These findings suggest that caution should be taken when considering cell therapy using BMMCs to treat PD. However, systemic BM-MSC transplantation that reaches the injury site and prevents neuronal damage after an MPTP infusion could be considered as a potential treatment for PD during the early stage of disease development.
\end{abstract}

Key words: MPTP; Bone marrow mononuclear cells; Mesenchymal stem cells; Parkinson's disease; Cell therapy. 


\section{Introduction}

Parkinson's disease (PD) is a chronic and slowly progressive neurodegenerative disorder that is classically characterized by resting tremors, bradykinesia, rigidity, postural instability and difficulty walking [1]. In addition to these motor symptoms, PD is characterized by non-motor symptoms, including neuropsychiatric symptoms, sleep disturbance, cognitive impairments and autonomic dysfunction [2,3].

PD results from a progressive loss of dopaminergic neurons in the substantia nigra pars compacta (SNpc), leading to reduced dopaminergic input to the striatum; this change is accompanied by the occurrence of Lewy bodies [1]. However, numerous studies have suggested that PD is a multiple neurotransmitter disorder that involves noradrenergic, cholinergic and serotoninergic systems in addition to the dopaminergic system [3,4].

The etiology and pathogenesis of idiopathic PD are still unknown. To elucidate the etiology and pathogenesis and to search the new treatments of PD, several animal models have been developed. Specifically, the 1-methyl-4-phenyl-1,2,3,6-tetrahydropyridine (MPTP) animal model is a useful tool because this model results in a dopaminergic loss in the SNpc that is associated with motor and cognitive impairment in rats similar to those observed in human PD $[5,6]$.

Studies into the pathogenesis of PD have suggested that many factors may be involved in the development of the disease, including aging, oxidative stress and neuroinflammation $[7,8]$. Several studies investigating MPTP have shown that the death mechanisms involved in the neurotoxicity of MPTP include the inhibition of complex I of the mitochondrial electron transport chain, the generation of reactive oxidative species (ROS) and neuroinflammation accompanied by a loss of integrity of the blood-brain barrier (BBB) $[9,10]$.

Although intensive efforts have been made in the search for new drugs to treat PD, current available therapeutic strategies treat only the motor symptoms, with little or no effects on non-motor symptoms and the progressive loss of dopaminergic neurons [11-13].

In recent years, multiple reports have suggested that transplantation with stem cells can provide therapeutic benefits in brain insult models of trauma, cerebral ischemia and exposure to neurotoxins [14-16].

The potential to replace dead neurons, restore the deficient neurotransmitters, reconstruct neuronal circuitries and provide neurotrophic factors to residual dopaminergic neurons in PD has emerged from several stem cells studies $[13,16,17]$. Different types of cell sources are considered useful for dopaminergic cell therapy in PD, including fetal ventral midbrain cells, embryonic stem cells, adult stem cells (e.g., bone marrow-derived cells) and induced pluripotent stem cells $[13,16,17]$. These cells have yielded promising results in both clinical and pre-clinical studies in PD [18-20]. However, clinical studies have shown inconsistent benefits in most transplanted PD patients. Moreover, future studies will need to address several obstacles, such as tumor formation, immune rejection and graft-induced dyskinesias, to ensure the safety and efficacy of this therapy $[17,21,22]$.

Adult bone marrow-derived cells, particularly mesenchymal stem cells (BM-MSCs), have received considerable attention in PD studies because of their relative feasibility and safety, the absence of religious and legal issues and the lack of immunological problems with autografts. These adult bone marrow stem cells are found in the mononuclear cell (BMMCs) fraction, which contains several progenitor white cells, including hematopoietic stem cells and endothelial progenitor cells $[23,24]$. These stem cells are described as multipotent cells that can be chemoattracted to the damaged tissue to release cytokines and trophic factors [25]. The use of BMMCs in comparison to purified stem cell therapies seems to be also promising, since this approach does not require gene manipulation or cell culture. Another advantage is the heterogeneity of the cell populations, which could guarantee an entire array of bone marrow-derived growth factors and cytokines that may also regulate cellular growth and regeneration via cellular secretion mechanisms [26]. Despite these arguments, there is no study in the literature in which the systemic administration of these BMMCs was performed in the context of PD.

The vast majority of the preclinical studies using stem cell therapy in PD have used MSCs locally injected into the SNpc or striatum. BM-MSCs comprise a small fraction of BMMCs (less than 0.01\%). These cells have been widely investigated and therapeutically used in pre-clinical studies in animal models of PD, showing some positive therapeutic benefits $[10,16,20,27-29]$. These studies have also shown that the transdifferentiation of BM-MSCs, also called bone marrow stromal cells (BMSCs), into neuronal cells remains unproven. Moreover, these cells have limited potential in cell replacement therapies for neurological disorders such as PD [29].

Although numerous studies have employed BM-MSCs as a perspective to treat the PD, there were no studies in the literature that compare the effect of BMMCs and BM-MSCs in the MPTP-rat model of PD. We hypothesized that systemic BMMC or BM-MSC therapy could be an alternative therapeutic strategy 
for PD that could delay neuronal damage in PD by blunting nigrostriatal pathway dysfunction [30].

Therefore, the main purpose of the present study was to determine the effects of BMMC and BM-MSC transplantation in MPTP-lesioned rats at different intervals after neurotoxin infusion. The effects were evaluated by behavioral tests and histological assays.

\section{Materials and methods}

\section{Animals}

All experiments were performed on Wistar rats obtained from the animal facility of the Department of Physiology of Triangulo Mineiro Federal University, Uberaba, MG. Male Wistar rats weighing 280-310 g at the beginning of the experiments were used. The rats were housed and maintained in a temperature-controlled room $\left(22 \pm 2^{\circ} \mathrm{C}\right)$ on a $12 \mathrm{~h}$ light-dark cycle (lights on at 7:00 am). The animals had free access to water and food throughout the experiment. All procedures adopted in the study were approved and conducted in according with the guidelines of the Committee on the Care and Use of Laboratory Animals Resources, Triangulo Mineiro Federal University, Uberaba, MG, Brazil.

\section{Stereotaxic surgery}

The MPTP and sham groups were anesthetized with sodium thiopental $(40 \mathrm{mg} / \mathrm{kg})$ and received a bilateral intranigral infusion of MPTP-chloride (100 $\mu \mathrm{g}$ in $1 \mu \mathrm{l}$ of saline) through a 30-gauge needle according to the following coordinates adapted from Paxinos and Watson's atlas: anteroposterior (AP), -5.0 $\mathrm{mm}$ from the bregma; mediolateral (ML), $\pm 2.1 \mathrm{~mm}$ from the midline; dorsoventral (DV), $8.0 \mathrm{~mm}$ from the skull [31]. Sham operations followed the same procedure but used $1 \mu \mathrm{l}$ of saline for the bilateral injection into the SNpc. After surgery, the animals were left in a temperature-controlled chamber until they had recovered from anesthesia.

\section{BMMC isolation and transplantation}

Bone marrow mononuclear cells (BMMCs) were obtained from normal donor Wistar rats. The animals were anesthetized with sodium thiopental $(40 \mathrm{mg} / \mathrm{kg}$, ip) and then euthanized. The femoral, tibial and humerus bones were collected, and the epiphyses were cut. After flushing the bones with a PBS solution, bone marrow cells were centrifuged for $10 \mathrm{~min}$ at $400 \times \mathrm{g}$, $4^{\circ} \mathrm{C}$. The pellet obtained after centrifugation was resuspended in sterile EDTA-PBS solution (pH 7.2), and the BMMC fraction was isolated using Ficoll-paque (GE Healthcare, Life Science) centrifugation procedures. The mononuclear cells were suspended in PBS-EDTA buffer, and the cell viability was tested using trypan blue $(0.4 \%)$. To study the identification and distribution of transplanted BMMCs in the brain injury site, cell labeling with a cell-surface marker DiI [1,1'-Dioctadecyl-3,3,3',3'-Tetramethylindocarbocyani ne] (CM-DiI, Molecular Probes Inc.) was performed in order to enable the identification of engrafted cells by fluorescence microscopy. All animals were injected with $1 \mathrm{ml}$ of saline or BMMC suspension (containing 5 $x 10^{6}$ cells) into the jugular vein immediately or $24 \mathrm{~h}$ after MPTP infusion (Experiments 1 and 2, respectively), according to the experimental design described below.

\section{BM-MSC isolation, culture and transplantation}

After isolating the BMMCs as described above, the cells were suspended in DMEM supplemented with $10 \%$ fetal bovine serum (FBS), $1 \%$ penicillin and streptomycin and seeded in culture bottles at a density of $4 \times 10^{5}$ cells $/ \mathrm{cm}^{2}$. After $48 \mathrm{~h}$ of incubation, the non-adherent cell population was removed by replacing the medium. When the adherent BM-MSC culture became confluent, the cells were detached with $0.25 \%$ trypsin, suspended in $1 \mathrm{mM}$ PBS-EDTA and then passaged. These procedures were repeated until the 4th passage. For studying the identification and distribution of transplanted MSCs in the brain injury site, the cells were labeled with a cell-surface marker as described above (CM-DiI, Molecular Probes Inc.). All animals were injected with $1 \mathrm{ml}$ of saline or BM-MSC suspension (containing $1 \times 10^{6}$ cells) into the jugular vein immediately after MPTP according to the experimental design described below (Experiment 1 ).

\section{Functional and phenotypic characterization of BM-MSCs}

To certify the stemness of BM-MSCs, these cells were submitted to a specific protocol to differentiate them into osteoblasts and adipocytes [32].

For the induction of osteogenic differentiation, BM-MSCs $\left(3,000\right.$ cells per $\left.\mathrm{cm}^{2}\right)$ were plated in culture dishes with the osteogenic induction medium consisting of DMEM plus 15\% FBS (fetal bovine serum), $1 \%$ penicillin/streptomycin, $100 \mathrm{nM}$ dexamethasone, $50 \mu \mathrm{M}$ ascorbate-2-phosphate and $10 \mathrm{mM}$ glycerol-phosphate. This medium was replaced every 3 days. On the 18th day, the analysis of differentiation was followed by the use of Alizarin Red (Sigma) to stain the bone matrix.

To induce adipogenic differentiation, BM-MSCs $\left(20,000\right.$ cells per $\left.\mathrm{cm}^{2}\right)$ were cultured in adipogenic induction medium consisting of DMEM plus $15 \%$ FBS, $1 \%$ penicillin/streptomycin, $1 \mu \mathrm{M}$ dexamethasone, $0.5 \mathrm{mM}$ isobutyl-methyl-xanthine, $10 \mu \mathrm{g} / \mathrm{ml}$ insulin and $100 \mu \mathrm{M}$ indomethacin. After 3 days, the adipogenic induction medium was replaced by adipogenic maintenance medium consisting of DMEM 
plus $15 \%$ FBS, $1 \%$ penicillin/streptomycin and 10 $\mu \mathrm{g} / \mathrm{m}$ insulin for $24 \mathrm{~h}$; at this time, the adipogenic maintenance medium was again replaced by adipogenic induction medium. After 3 cycles of media changes $(72 \mathrm{~h}$ with induction medium and $24 \mathrm{~h}$ with maintenance medium), the cells were maintained with the maintenance medium for 5 days, and the analysis of differentiation was performed using Oil-Red to stain the lipid droplets.

In addition, a phenotypic characterization of BM-MSCs was performed by quantifying the cell membrane markers by flow cytometry. The following markers were investigated: CD11b, c-Kit, CD45, CD29, CD34 and CD31 antibodies (BD Biosciences Inc., San Jose, CA, USA; e-Biosciences, San Diego, CA, USA).

\section{Experimental design}

\section{Experiment I:}

This experiment was performed with aim to verify the effects of the systemic transplantation of BMMCs and BM-MSCs on the progression of MPTP-induced lesion. For this, the animals were transplanted with BMMCs or BM-MSCs immediately after bilateral intranigral MPTP infusion and then, evaluated on motor function by the open field test and on the quantity of positive tyrosine hydroxylase (TH) neurons in the SNpc. The animals were randomly divided into six groups ( $\mathrm{n}=8-12$ animals/group): Sham-saline, Sham-BMMC, Sham-BM-MSC, MPTP-saline, MPTP-BMMC and MPTP-BM-MSC groups. After stereotaxic surgery, the animals were intravenously injected with $5 \times 10^{6} \mathrm{BMMC} / \mathrm{ml}$ labeled with CM-DiI, $1 \times 10^{6} \mathrm{BM}-\mathrm{MSC} / \mathrm{ml}$ labeled with CM-DiI or saline. Twenty-four hours after stereotaxic surgery, the animals were exposed to the open field test to determine motor alterations. After this test, the animals were intracardially perfused with saline and fixative solution (formaldehyde $4 \%$ in $0.1 \mathrm{M}$ phosphate buffer, $\mathrm{pH}$ 7.4). The brain was removed from the skull and was immersed in the same fixative solution overnight. Next, the brain was placed in 30\% sucrose solution for $48 \mathrm{~h}$ before the freezing procedure and was stored until analysis. Figure $2 \mathrm{~A}$ describes the time course of Experiment 1.

\section{Experiment 2:}

This experiment was performed to verify the effects of transplantation of BMMCs when the MPTP-induced lesion was installed. For this, the animals were transplanted with BMMCs $24 \mathrm{~h}$ after bilateral intranigral MPTP infusion and then evaluated on motor function using the open field test, on behavior function analyzed by the forced swimming test and on the number of TH-labeled neurons in the SNpc quantified by immunohistochemistry. The animals were randomly divided into 4 groups ( $\mathrm{n}=8-12$ animals/group): Sham-saline, Sham-BMMC, MPTPsaline and MPTP-BMMC groups. Twenty-four hours after stereotaxic surgery, the animals were submitted to the open field test to determine motor alterations. After this test, the animals were intravenously injected with $5 \times 10^{6} \mathrm{BMMC} / \mathrm{ml}$ labeled with CM-DiI or saline. The open field test was repeated 3 and 7 days after surgery. Seven days after stereotaxic surgery, the animals were also gently exposed to the forced swimming test applied in two steps on the 7th and 8th days after MPTP injection. After completing the tests, the animals were intracardially perfused as described for Experiment 1. Figure 3A describes the time course for Experiment 2.

\section{General motor activity test}

The general motor activity test was conducted in a white open-field arena constructed according to Broadhurst [33]. Briefly, the apparatus testing was a circular arena with a diameter of $97 \mathrm{~cm}$, whose floor was divided by dark lines creating equal sized areas. The rats were placed individually in the center of the apparatus and observed for $5 \mathrm{~min}$. The number of lines crossed (locomotion frequency) and rearing frequency (number of times the animals stood on their hind paws) was determined. Stopwatches were used to score immobility time (time of no movement during testing) and latency to start the movement (latency time to leave the first circle) which was used to evaluate akinesia [34]. In order to eliminate possible bias due to odors left by previous rats, the open field was washed with $5 \%$ water-ethanol solution before each the motor activity test

\section{Forced swimming test}

Despair induced by the forced swimming test is widely used as a preclinical model of depression [35]. Swimming sessions were conducted by placing rats in individual PVC cylinders $(46 \mathrm{~cm}$ tall $\times 20 \mathrm{~cm}$ in diameter) containing $23-25^{\circ} \mathrm{C}$ water at a depth of $30 \mathrm{~cm}$ so that the rats could not support themselves by touching the bottom with their paws and tail. Two swimming sessions were conducted: 1 ) a pretest, performed for 15 min 7 days after surgery, and 2) the test, performed $24 \mathrm{~h}$ after the pretest session (Figure 3A). During the test session ( $5 \mathrm{~min}$ ), the following parameters were observed and measured in seconds: climbing time (during which the animal performs active movements with forepaws in and out of the water, usually directed against the walls), swimming time (during which the animal performs active swimming motions between quadrants of the cylinder, more than necessary to merely keep the head 
above water) and immobility time (during which the animal floats in the water without struggling, making only those movements necessary to keep its head above the water). Following both swimming sessions, the rats were removed from the water, dried and placed in heated cages. Test sessions were video-taped from above the cylinders for further evaluation. The water in the cylinders was replaced after every trial.

\section{Tyrosine hydroxylase (TH) immunohisto- chemistry}

Immediately after the motor activity or behavioral test, the animals were deeply anesthetized with sodium thiopental $(100 \mathrm{mg} / \mathrm{kg}$, ip) and intracardially perfused with $4 \%$ paraformaldehyde in $0.1 \mathrm{M}$ phosphate buffer, $\mathrm{pH}$ 7.4. The brain was dissected from the skull and postfixed in the fixative solution for 1 week at $4^{\circ} \mathrm{C}$. Before sectioning, the brain was placed in $30 \%$ sucrose solution for $48 \mathrm{~h}$ and then stored in a freezer $\left(-80^{\circ} \mathrm{C}\right)$. Brains were cut in the coronal plane in twelve series of $20-\mu \mathrm{m}$ thick sections using a cryostat. The sections were washed with PBS and incubated with primary anti-TH antibody raised in rabbits and diluted in PBS containing 0.3\% Triton X-100 (1:500; Chemicon, Temecula, CA, USA) overnight at $4^{\circ} \mathrm{C}$. The slides were then incubated with the biotin-conjugated secondary antibody (1:200, Vector Laboratories, Burlingame, CA, USA) for $2 \mathrm{~h}$ at room temperature. The sections were washed in PBS and the antibody complex was detected using a modified $\mathrm{ABC}$ system (Vectastain ABC Elite kit, Vector Laboratories), followed by reaction with 3,3'-diaminobenzidine (DAB) with nickel enhancement. The severity of neuronal damage in the midbrain due to MPTP was stereologically quantified. First, TH-immunorreactive (TH-ir) neurons in the SNpc of control animals were quantified, which showed a normal $\mathrm{TH}$ immunostaining. After, the same parts of the dopaminergic cell groups in the MPTP groups were measured. For this approach, selected areas in a 10x microscope magnification per section were digitized using a DP71 Olympus Optical digital camera with a BX50 Olympus microscope. The digital images transferred to a computer were analyzed by means of detection of optical density of each cut using the software "Image J". The optical density value from the lesioned region was expressed as a percentage of the control group on the same section.

\section{$B M M C$ and $B M-M S C$ tracking in recipient rat brains}

Twenty-four hours and 7 days after cellular transplantation, rats were intracardially perfused and the brain was removed and frozen. The samples were cut in a cryostat (Leica CM 1850 UV), and tissue sections were collected and incubated with 4',6-diamidine-2-phenylindole (DAPI) counterstain solution (Sigma) (1:10000 dilution) for $5 \mathrm{~min}$ at room temperature. The slices were carefully rinsed in PBS and analyzed under a fluorescence microscope (AxioObserver Z1, Carl Zeiss, Germany) to identify the BMMCs and BM-MSCs labeled with CM-DiI and DAPI.

\section{Immunohistochemistry for CD45}

In another set of animals, in which BMMCs and BM-MSCs were not previously labeled with the DiI-CM cell tracker, the in situ expression of CD45, a marker of white blood cells, was examined in brain sections obtained from perfused animals as described above. Tissue samples were incubated in blocking solution containing 5\% normal goat serum (Sigma Chemical Co.) and 3\% BSA in 0.1 M PBS for $2 \mathrm{~h}$ at room temperature. After this period, brain sections were incubated with monoclonal mouse anti-rat CD45 labeled with phycoerythrin (PE) (BD Pharmigen) (1:20 dilution in PBS containing 5\% normal goat serum and $3 \% \mathrm{BSA}$ ) overnight at $4^{\circ} \mathrm{C}$. After several washes in PBS, the brain sections were incubated with 4',6-diamidine-2-phenylindole (DAPI) counterstain solution (Sigma) (1:10000 dilution) for $5 \mathrm{~min}$ at room temperature. The slices were carefully rinsed in PBS and analyzed under a fluorescence microscope (AxioObserver Z1, Carl Zeiss, Germany). Control experiments were performed by omitting the primary antibody.

\section{Immunohistochemistry for Iba I (Ionized calcium binding adaptor molecule I)}

In order to investigate the possible microglial activation after BMMC transplantation, midbrain sections from another set of animals were also immune stained with an antibody against ionized calcium binding adaptor molecule 1(Iba1), an widely used marker of microglia [36,37].

The tissue processing for immunohistochemistry was conducted as described in TH-immunohistochemistry item. Briefly, after anesthesia with sodium thiopental $(100 \mathrm{mg} / \mathrm{kg}$, ip), the animals were euthanized and intracardially perfused with saline followed by buffered $4 \%$ paraformaldehyde solution, $\mathrm{pH}$ 7.4. The brain was carefully excised and cryopreserved in $30 \%$ sucrose in PBS for 48 hours and then frozen at $-80^{\circ} \mathrm{C}$ until sectioning and immunohistochemical processing. Twelve series of $20-\mu \mathrm{m}$ thick coronal sections of midbrain were obtained using a cryostat as describe above. Following an initial heating in a microwave oven for six minutes and cooling in room temperature for 15 minutes, all tissue sections 
were blocked with $2.5 \%$ normal horse serum and incubated with polyclonal goat anti-Iba1 antibody (1:500, Abcam) diluted in PBS containing 0.3\% Triton $\mathrm{X}-100$ overnight at $4^{\circ} \mathrm{C}$. The slides were then incubated with the biotin-conjugated secondary antibody (1:200, Vector Laboratories, Burlingame, CA, USA) for $2 \mathrm{~h}$ at room temperature. After rinsing in PBS, the immunohistochemical reaction was performed using $\mathrm{DAB}$ and the sections were dehydrated through graded ethanol, cleared in xylene, and coverslipped in mounting medium. For analysis, morphological features of microglia from SNpc and ventral tegmental area (VTA), used as a non-injured site control, were analyzed per section under a microscope (AxioObserver Z1, Carl Zeiss, Germany). Control reaction experiments were performed by omitting the primary antibody.

\section{BBB permeability assay}

Evans blue (EB, Sigma) is a low-molecularweight dye commonly used to determine BBB disruption. Evans blue binds to serum albumin $(65 \mathrm{kDa})$ and only crosses the BBB if there is an increase in $\mathrm{BBB}$ permeability. Therefore, the extravasation of $\mathrm{EB}$ is indicative of BBB breakdown [38]. For this assay, another set of animals was submitted to stereotaxic surgery as described above (stereotaxic surgery section). However, in this case, the animals received a unilateral MPTP-HCl infusion (100 $\mu \mathrm{g}$ in $1 \mu \mathrm{l}$ of saline) and saline solution in the contralateral side. Changes in BBB permeability were investigated at two intervals after MPTP infusion: $24 \mathrm{~h}$ and 7 days after MPTP infusion. The first group ( $24 \mathrm{~h}$ after surgery) was anesthetized with sodium thiopental $(40 \mathrm{mg} / \mathrm{kg}$, ip) and injected in the jugular vein with EB $2 \%(4 \mathrm{ml} / \mathrm{kg}$ of body weight). The stain was allowed to circulate for $30 \mathrm{~min}$. The animals then were intracardially perfused with saline, and the same fixing and sectioning procedures described above were used. The location of extravasated EB on the $10-\mu \mathrm{m}$ coronal section of SNpc was observed with a fluorescent microscope (Axi-
oObserver Z1, Carl Zeiss, Germany) with an excitation wavelength of 530-550 $\mathrm{nm}$ and an emission wavelength of $>590 \mathrm{~nm}$. On the contralateral side, the autofluorescence was recorded with the same excitation/emission parameters. A second group of animals was submitted to the same procedure. However, these animals were injected with the EB probe 7 days after unilateral MPTP infusion and were intracardially perfused $30 \mathrm{~min}$ after this injection.

\section{Statistical analysis}

Parametric data were analyzed by one-way ANOVA followed by the Tukey-Kramer Test. The Mann-Whitney U-Test or the Kruskal-Wallis Test (KW) followed by Dunn's multiple comparison tests was used to analyze non-parametric data. All values are expressed as the mean \pm SEM.

\section{Results}

\section{Functional and phenotypic characterization of BM-MSCs}

After the isolation and culture of the BM-MSCs, the cells grew to confluence and exhibited morphology similar to fibroblasts (Figure 1A). When the BM-MSCs were submitted to adipogenic stimulation, a shift in cell morphological features was observed from a primarily slender morphology to a spherical cell phenotype, and these cells were positively stained for lipid vacuoles by Oil Red-O (Figure 1B). In contrast, BM-MSC culture submitted to the osteogenic differentiation protocol resulted in an extracellular matrix positively stained for Alizarin Red (Figure 1C).

Cultured BM-MSCs submitted to phenotypic characterization by means of flow cytometry exhibited the following profile: CD11b (7.83\%), CD34 (8.67\%), CD45 (15.80\%), CD31 (2.47\%), c-Kit (8.35\%) and CD29 $(89.64 \%)$. These findings indicated a reduced number of contaminating cells such as macrophages, hematopoietic stem cells, leukocytes and endothelial cells.
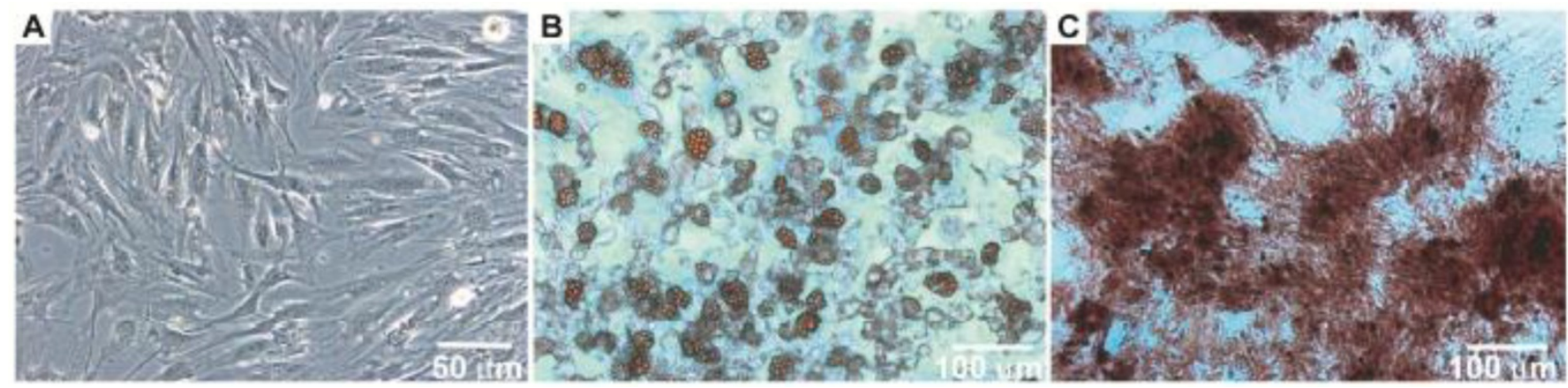

Figure I. Morphology of bone marrow MSC (Panel A). Photomicrographs illustrating BM-MSC differentiation under adipogenic (Panel B) and osteogenic (Panel C) differentiation-inducing conditions. 


\section{The effect of BMMC and BM-MSC transplantation in MPTP-lesioned rats on the general motor activity evaluated by the open field test: Experiment I}

The effect of BMMC and BM-MSC transplantation immediately after MPTP infusion on the open field test is shown in Figures 2B-2E. The MPTP-saline group exhibited a significantly increased immobility time $(\mathrm{P}<0.05)$ compared to the Sham-saline group and decreased rearing frequencies $(P<0.05)$ compared to the Sham-saline and Sham-BMMC groups. Furthermore, the MPTP-saline group showed increased latency to initiate movement $(\mathrm{P}<0.05)$ compared to the Sham-BMMC group. The MPTP-lesioned rats transplanted with BMMCs showed decreased rearing frequencies $(\mathrm{P}<0.05)$ and increased immobility time $(\mathrm{P}<0.05)$ compared to both Sham groups. Moreover, the MPTP-BMMC group exhibited increased latency to initiate movement $(\mathrm{P}<0.05)$ compared to the Sham-BMMC group and decreased locomotion frequency compared to the Sham-BMMC (P $<0.01)$ and Sham-BM-MSC $(\mathrm{P}<0.05)$ groups. There were no differences in any open field parameter when the MPTP-Saline and MPTP-BMMC groups were compared. In addition, no differences were found between the MPTP-saline and MPTP-BM-MSC groups for any of the open field parameters.

A.

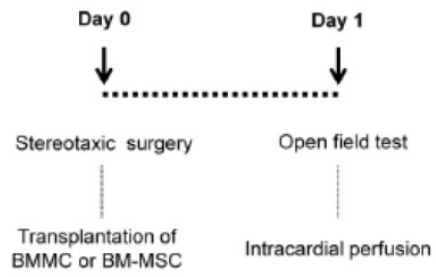

B.

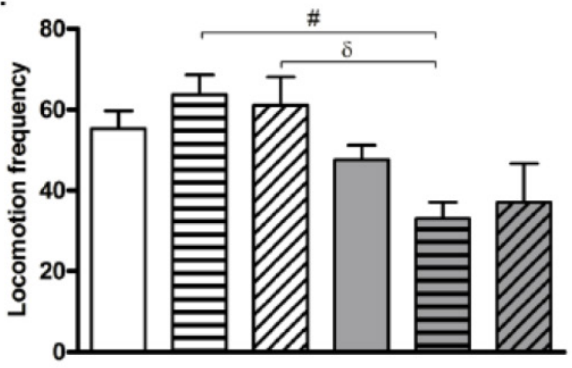

D.

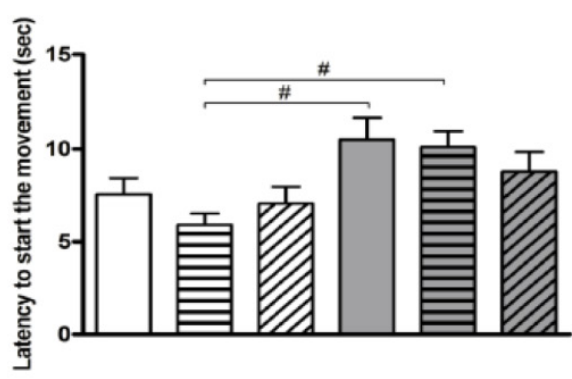

C.

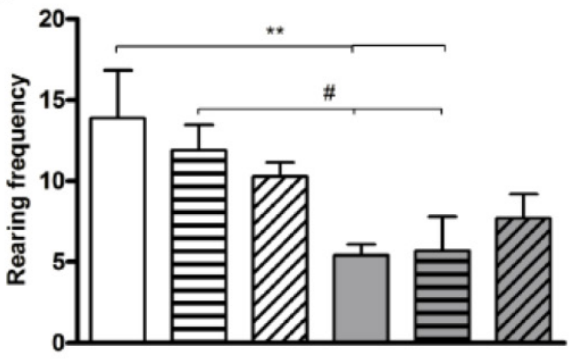

E.

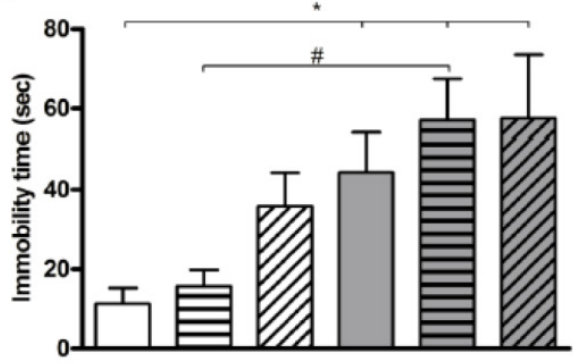

Figure 2. A) Time course Experiment I. B-E) The effect of BMMC and BM-MSC transplantation immediately after MPTP infusion on the open field test $24 \mathrm{~h}$ after surgery in the following groups: Sham-saline $(n=8)$ Sham-BMMC $(n=12)$ Sham-BM-MSC $(n=9)$, MPTP-saline $(n=10)$ MPTP-BMMC $(n=10)$ and MPTP-BM-MSC ( $=9)$. Values are the mean \pm SEM. *P < 0.05 and $* * P<$ 0.0I: significantly different from the Sham-saline group; \#P < 0.05: significantly different from the Sham-BMMC group; and $\delta P<0.05$ : significantly different from the Sham-BM-MSC group. A one-way ANOVA followed by Tukey-Kramer multiple comparison test or Kruskal-Wallis test followed by Dunn's multiple comparison test was used to analyze the data. 


\section{The effects of BMMC transplantation in MPTP-lesioned rats on the general motor ac- tivity evaluated by the open field test: Exper- iment 2}

The data illustrated in Figures 3B-3E show that the administration of MPTP caused a significant reduction in the general motor activity of rats, as measured by the open field test $24 \mathrm{~h}$ after stereotaxic surgery and before BMMC transplantation.

In the first $24 \mathrm{~h}$, MPTP-lesioned rats presented a significant decrease in locomotion frequency $(\mathrm{P}<$
$0.0001)$ and rearing frequency $(\mathrm{P}=0.0011)$ compared to the Sham group. Furthermore, the MPTP group exhibited a significant increase in immobility time ( $P$ $=0.0003)$ and latency to start the movement time $(\mathrm{P}=$ 0.029) compared to the Sham group. Except for latency to initiate movement, which remained increased after $3(\mathrm{P}<0.05)$ and 7 days $(\mathrm{P}<0.05)$ post-lesion, the other parameters were completely recovered to levels similar to the levels of the control animals (Sham-saline group).

A.

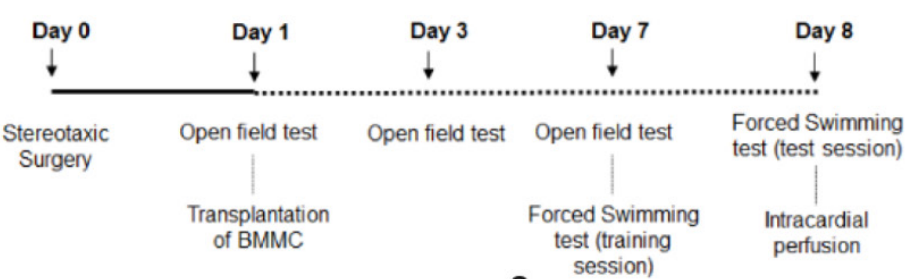

B.

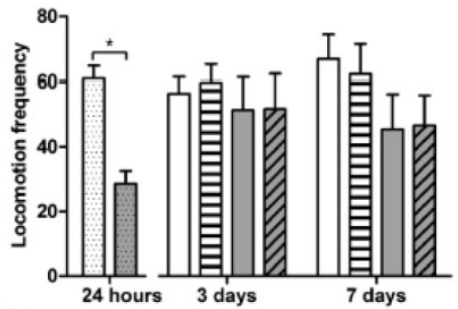

D.

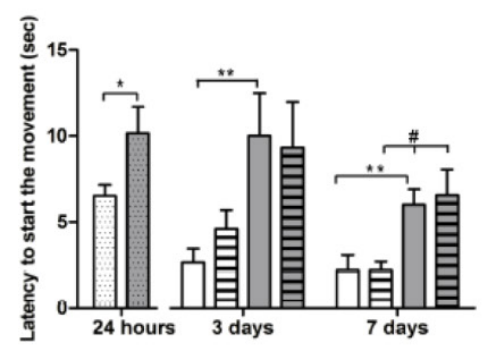

F.

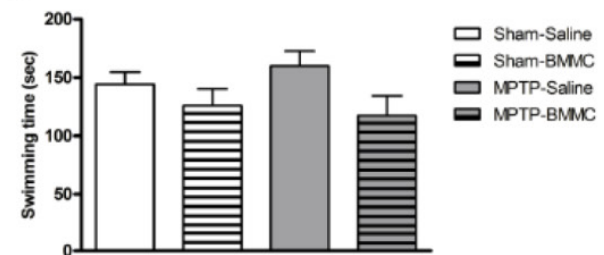

H.

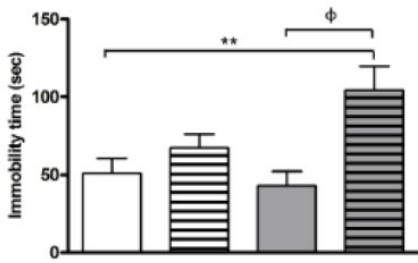

Sham 四 MPTP 口 Sham-Saline Sham-BMMC $\square$ MPTP-Saline Z MPTP-BMMC

c.

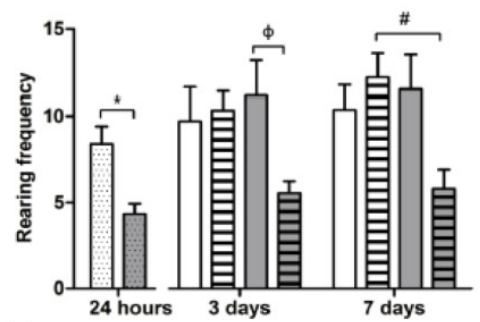

E.

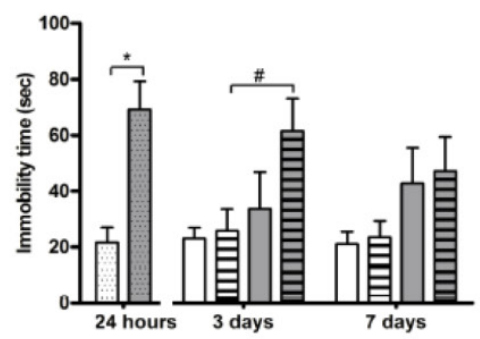

G.

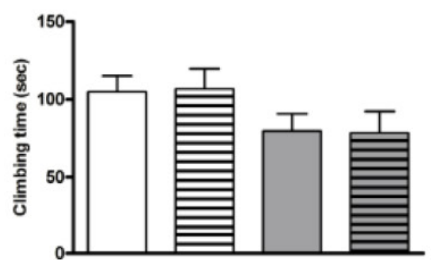

Figure 3. A) Time course of Experiment 2. B-E) The effect of MPTP infusion and BMMC transplantation on the general activity assessed by the open field test $24 \mathrm{~h}, 3$ days and 7 days after surgery in the following groups: Sham $(n=19)$ and MPTP $(n=20)$, Sham-saline $(n=8)$, Sham-BMMC $(n=10)$, MPTP-saline $(n=8)$, MPTP-BMMC $(n=10)$. F-H) The effect of BMMC transplantation and MPTP infusion on the forced swimming test 8 days after stereotaxic surgery in the following groups: Sham-saline $(n=7)$, Sham-BMMC ( $n=9$ ), MPTP-saline $(n=7)$, MPTP-BMMC ( $=9$ ). Values are the mean + SEM $*$ P $<0.01$ : significantly different from the Sham group $24 \mathrm{~h}$ after surgery; **P $<0.05$. significantly different from the Sham-saline group three days and seven days after MPTP infusion; \#P < 0.05: significantly different from the Sham-BMMC group; and $\Phi \mathrm{P}<0.05$ : significantly different from the MPTP-Saline group. The Student $t$-test or Mann-Whitney U-test was performed to analyze the open field test data collected $24 \mathrm{~h}$ after MPTP infusion. A one-way ANOVA followed by Tukey-Kramer multiple comparison test or Kruskal-Wallis test followed by Dunn's multiple comparison test was performed to analyze the data from the forced swimming test 8 days after surgery and the open field test 3 and 7 days after surgery. 
The effect of BMMC transplantation on the open field test 3 and 7 days after MPTP infusion is shown in Figures 3B-3E. Three days after stereotaxic surgery, MPTP-lesioned rats that received BMMC showed a significant increase in immobility time $(\mathrm{P}<0.05)$ compared to the Sham-BMMC group and decreased rearing frequency compared to the MPTP-saline group $(\mathrm{P}<0.05)$. There were no differences in the other parameters among the experimental groups 3 days after stereotaxic surgery. Seven days after surgery, the MPTP-BMMC group presented decreased rearing frequency $(\mathrm{P}<0.05)$ and increased latency to initiate movement $(\mathrm{P}<0.05)$ compared to the Sham-BMMC group. No differences in the other parameters were observed among experimental groups 7 days after stereotaxic surgery.

\section{Effects of BMMC transplantation in MPTP-lesioned rats on behavior evaluated by the forced swimming test}

The results depicted in Figures 3F-3H show the effect of MPTP infusion and BMMC transplantation 8 days after stereotaxic surgery in the rats. There were no differences in swimming and climbing time among the groups tested. However, the MPTP-lesioned rats
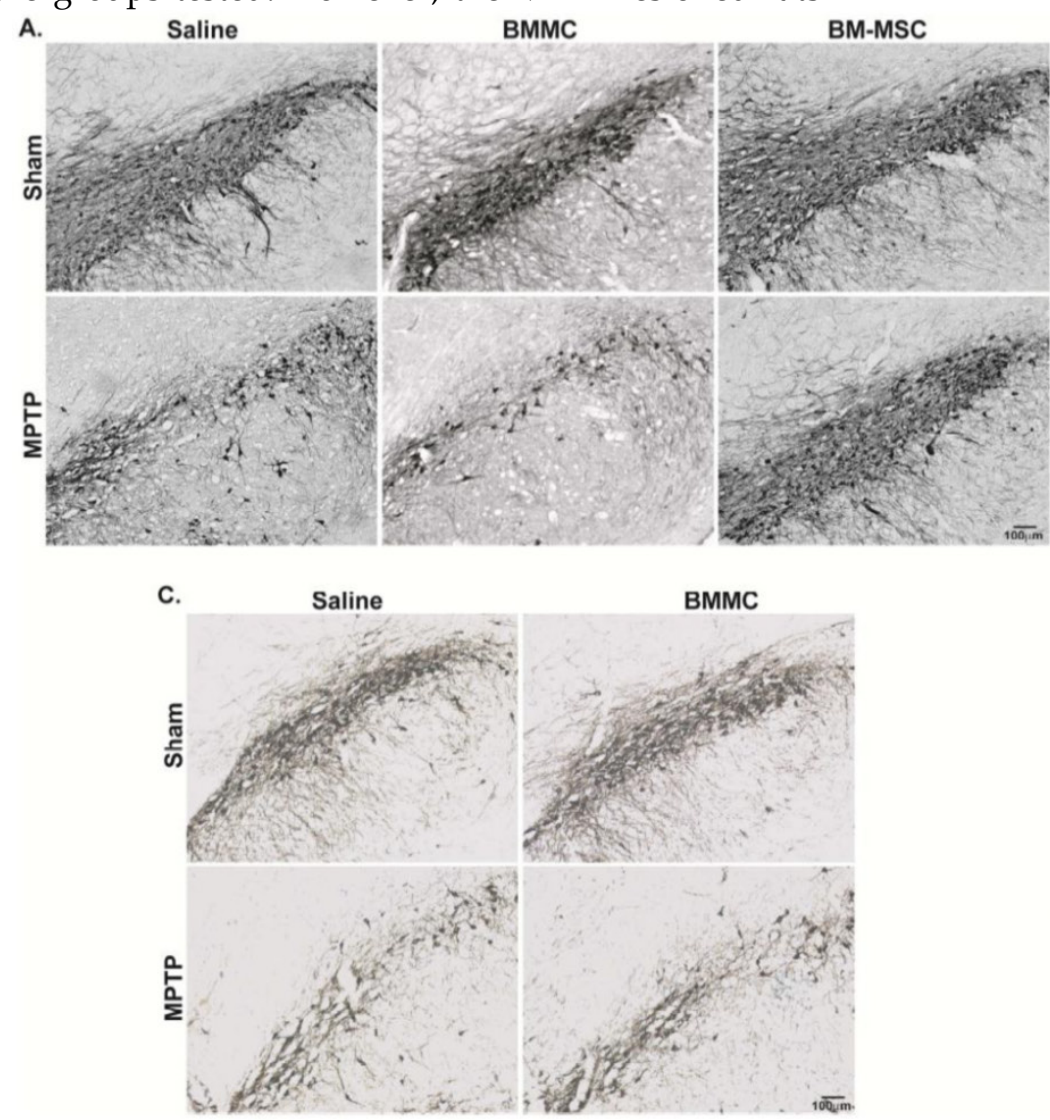

Figure 4. Photomicrography of representative sections of tyrosine hydroxylase-immunoreactive (TH-ir) neurons in the substantia nigra pars compacta (SNpc) $24 \mathrm{~h}$ (Panel A) and 8 days (Panel C) after MPTP injection. Scale bar: $100 \mu \mathrm{m}$. C) The effect of BMMC and BM-MSC transplantation in the MPTP model of Parkinson's disease on the percent loss of TH-ir neurons in the SNpc of rats $24 \mathrm{~h}$ after MPTP injection compared to Sham groups ( $\mathrm{n}=5$ animals/group). D) The effect of MPTP infusion and BMMC transplantation on the percent loss of TH-ir neurons in the SNpc of rats 8 days after MPTP injection compared to Sham groups $(n=5$ animals/group). Values are the mean \pm SEM. $* P<0.01$ compared to the Sham-saline group; \#P $<0.01$ compared to the Sham-BMMC group, $\delta \mathrm{P}<0.01$ compared to the Sham-BM-MSC group, $\Phi \mathrm{P}<0.01$ compared to the MPTP-Saline group and $\alpha \mathrm{P}<0.00 \mathrm{I}$ compared to the MPTP-BMMC group. A Kruskal-Wallis test followed by Dunn's multiple comparison test was used to analyze the data. transplanted with BMMCs $24 \mathrm{~h}$ after MPTP injection showed a significant increase in immobility time $(\mathrm{P}=$ $0.0047)$ compared to the Sham-saline $(\mathrm{P}<0.05)$ and MPTP-saline groups $(\mathrm{P}<0.01)$.

\section{TH immunohistochemistry}

The administration of MPTP caused significant TH-ir cells loss in the SNpc $24 \mathrm{~h}$ (Figures 4A and 4B) and 8 days (Figures $4 \mathrm{C}$ and $4 \mathrm{D}$ ) after stereotaxic surgery ( $\mathrm{P}<0.01$ and $\mathrm{P}<0.001$, respectively) compared to the Sham groups. The MPTP-saline group exhibited approximately $20 \%$ and $38 \%$ TH-ir cells loss $24 \mathrm{~h}$ and 8 days after MPTP injection, respectively, compared to the Sham-saline group. Twenty-four hours after MPTP injection, the MPTP-BMMC group exhibited significantly increased TH-ir cells loss compared to the Sham-saline $(\mathrm{P}<0.001)$, Sham-BMMC $(\mathrm{P}<0.01)$, Sham-BM-MSC $(\mathrm{P}<0.001)$ and MPTP-saline $(\mathrm{P}<0.01)$ groups. However, the MPTP-lesioned rats transplanted with BM-MSCs did not exhibit a loss of TH-ir cells in the SNpc compared to the MPTP-BMMC group $(P<0.001)$, and these animals presented no difference compared to the Sham groups.

B.

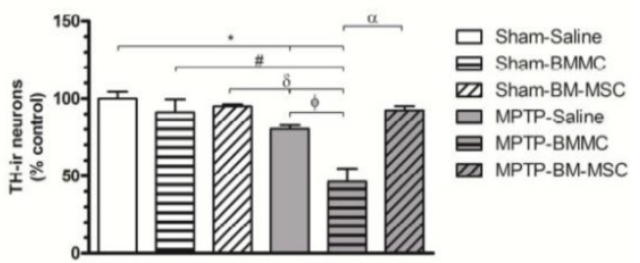

D.

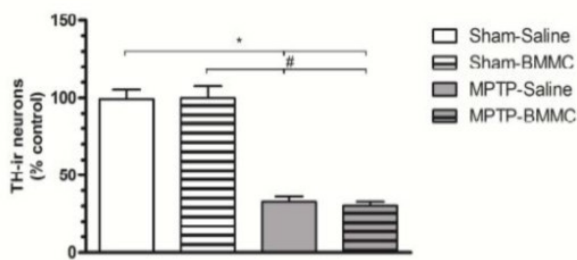


A.

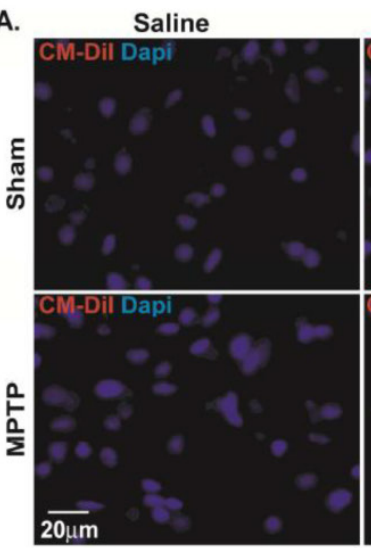

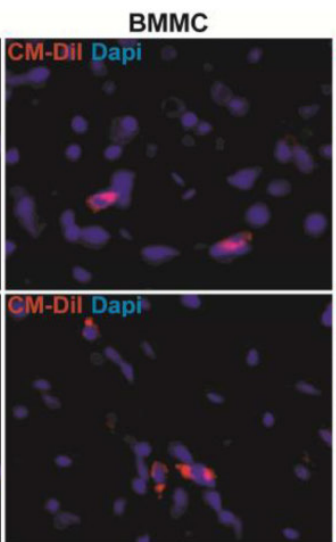

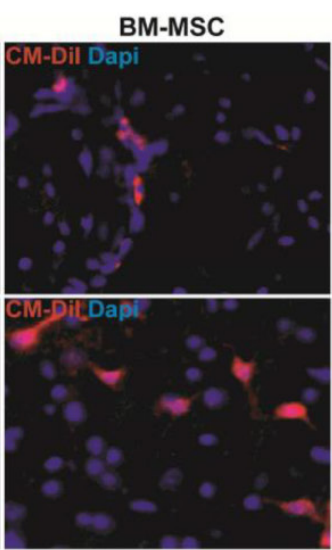

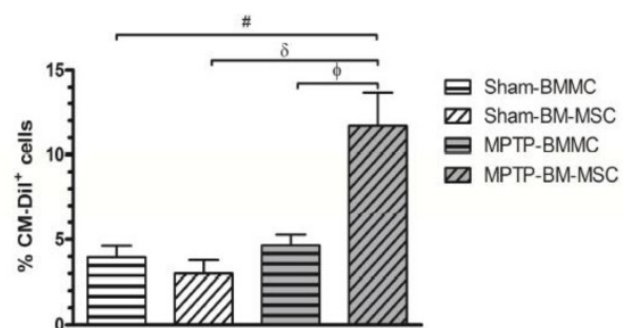

D.

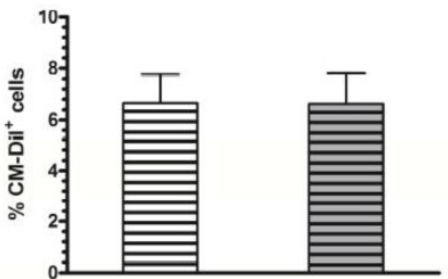

曰 Sham-BMMC 曰 MPTP-BMMC
B.

MPTP-BM-MS
C.

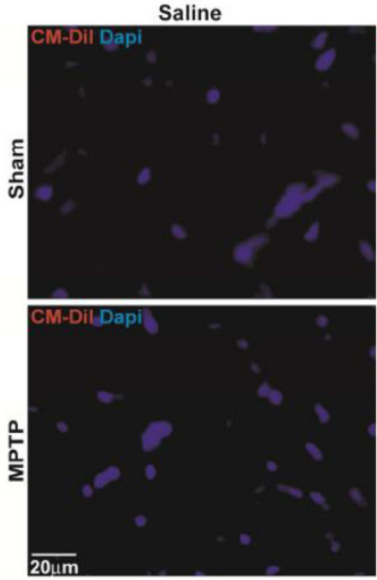

BMMC

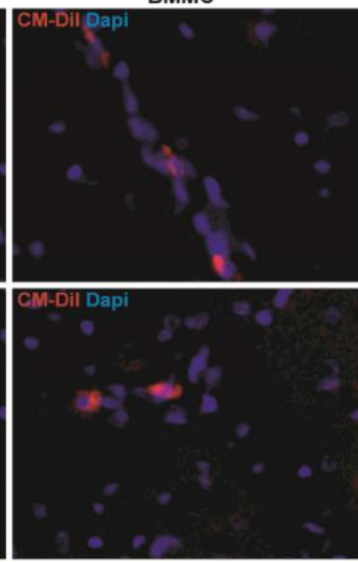

Figure 5. Photomicrography illustrating BMMCs and BM-MSCs labeled with CM-Dil. These cells were found in the brain samples of transplanted animals $24 \mathrm{~h}$ (Panel A) and 8 days (Panel C) after stereotaxic surgery. B) Percentage of BMMC and BM-MSC labelled with CM-Dil founded 24 hours after MPTP administration. D) Percentage of BMMC labelled with CM-Dil founded 8 days after MPTP administration. $\mathrm{N}=4$ animals/group. Scale bar: $20 \mu \mathrm{m}$.

No difference in the TH-immunoreactivity loss 8 days after surgery was observed between the MPTP-saline and MPTP-BMMC groups.

\section{BMMC and BM-MSC identification in the brain samples of recipient rats}

Twenty-four hours (Figures $5 \mathrm{~A}$ and $5 \mathrm{~B}$ ) and 7 days (Figures 5C and 5D) after cellular transplantation, CM-DiI-labeled cells were found in all SNpc sections of the Sham-BMMC, Sham-BM-MSC, MPTP-BMMC and MPTP-BM-MSC groups. The number of $\mathrm{CM}_{-}-\mathrm{DiI}^{+}$cells in the SNPC from the MPTP-BM-MSC animals $(11.69 \pm 1.96 \%, \mathrm{P}<0.05)$ was significantly higher than the numbers observed in the other groups of experiment $1 \quad(3.95 \pm 0.62 \%$ for Sham-BMMC, $3.00 \pm 0.80 \%$ for Sham-BM-MSC and $4.64 \pm 0.63 \%$ for MPTP-BMMC animals). No statistic difference was observed between the studied groups at number of labeled cell in the experiment 2. As expected, there was no identification of labeled cells in the Sham-saline and MPTP-saline groups in the both experiments.

\section{Expression of CD45 in the rat brain slices}

Figures $6 \mathrm{~A}$ and $6 \mathrm{~B}$ shows the expression of CD45 and DAPI in the brain samples of all animal groups at $24 \mathrm{~h}$ after stereotaxic surgery. Figures $6 \mathrm{C}$ and $6 \mathrm{D}$ shows the expression of CD45 and DAPI in the brain slice of all animal groups studied at 7 days after transplant of cells. MPTP-lesioned rats transplanted with BMMCs immediately after lesioning showed an increased quantity of $\mathrm{CD} 45^{+}$cells $(7.43 \pm 0.93 \%)$ compared to the Sham-saline $(2.54 \pm 0.34 \%, \mathrm{P}<0.05)$, Sham-BMMC $(3.38 \pm 0.48 \%, \mathrm{P}=0.01)$, Sham-BM-MSC (1.44 $\pm 0.09 \%, \mathrm{P}<0.05)$, MPTP-saline $(3.04 \pm 0.27 \%, \mathrm{P}<$ $0.01)$ and MPTP-MSC $(2.87 \pm 0.73 \%, \mathrm{P}<0.05)$ groups. Moreover, 8 days after stereotaxic surgery, the MPTP-lesioned rats that received BMMC administration $24 \mathrm{~h}$ after neurotoxin infusion exhibited an increased quantity of $\mathrm{CD}_{4} 5^{+}$cells $(4.61 \pm 0.50 \%)$ compared to the Sham-saline $(2.88 \pm 0.38 \%, \mathrm{P}<0.037)$ and Sham-BMMC $(3.08 \pm 0.34 \%, \mathrm{P}<0.058)$ groups. No differences were observed among the other studied groups. 

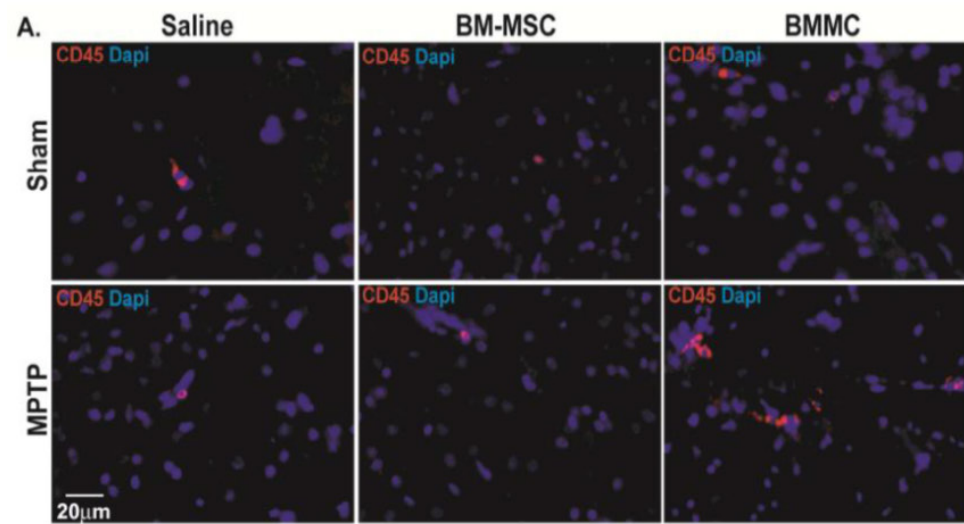

C.
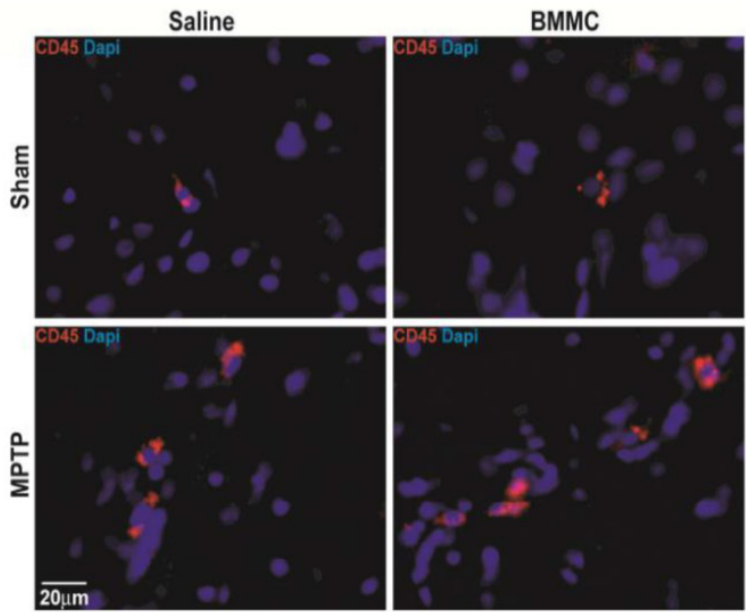

B.

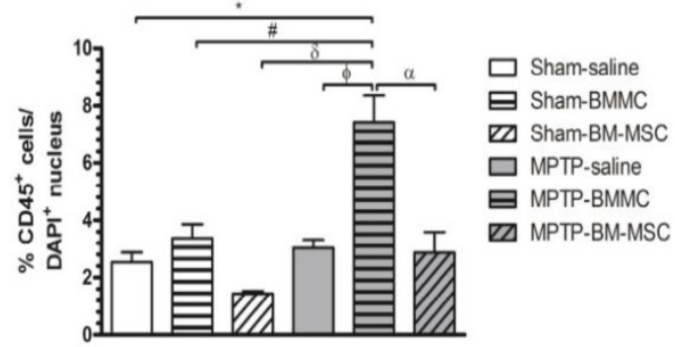

D.

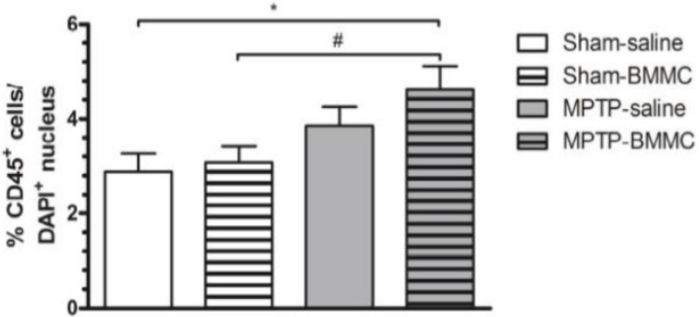

Figure 6. Immunohistochemical analyses for CD45 expression in the rat brain slices $24 \mathrm{~h}$ (Panel A) and 8 days (Panel C) after stereotaxic surgery. B) Percentage of CD45 expression founded $24 \mathrm{~h}$ after MPTP administration. D) Percentage of CD expression founded 8 days after MPTP administration. N=4 animals/group. Scale bar: $20 \mu \mathrm{m}$.

\section{Ibal immunohistochemistry}

Microglial cells were visualized in tissue sections by immunohistochemical labeling of the Iba1 antigen in both areas of midbrain studied, SNpc and VTA, in all selected Sham-saline, Sham-BMMC, MPTP-saline and MPTP-BMMC groups (Figure 7B-J). No evident differences were found in the VTA among the all studied groups and one picture was choose to illustrate the morphology of microglial cells in the VTA (Figure 7B). However, in the SNpc, site of MPTP infusion, there was pronounced change of microglial morphology from resting state, which is characterized by many fine processes and a small cell body, to a reactive state characterized by bushy, hyper-ramified or amoeboid morphology (Figure 7G-J). In addition, the presence of activated microglial cells was more abundant in MPTP-lesioned rats transplanted with BMMC immediately after lesion than animals lesioned with MPTP and treated with saline (Figure 7I).

\section{The effect of MPTP infusion on BBB integrity at $24 h$ and 7 days}

An analysis of cross-sections of the brains after the injection of the EB dye in unilateral
MPTP-lesioned rats at the SNpc demonstrated that MPTP injury resulted in significant EB extravasation into the parenchyma at the injury site. This finding indicates the severe BBB breakdown at both the 24-h and 7-day intervals. There was no EB extravasation into the parenchyma at the contralateral injury site, which saline was injected demonstrating BBB preservation at both intervals (Figure 8).

\section{Discussion}

The present study showed that systemic BMMC transplantation did not ameliorate or prevent the lesion induced by MPTP in rats. Instead, this BMMC transplantation in MPTP-lesioned accelerated the dopaminergic neuronal damage and induced the immobility behavior. In contrast, systemic BM-MSC transplantation was able to prevent dopaminergic neuronal damage in the SNpc despite the minor functional improvements in motor activity.

Our results showed that MPTP injection produced a significant reduction in locomotion and rearing frequencies after $24 \mathrm{~h}$ as assessed by the open field test. Moreover, these rats exhibited a significant increase in the immobility time and in the latency to initiate movement. These general motor activity al- 
terations are important features of this MPTP model of PD and have been demonstrated in many studies $[5,39]$. Three and 7 days after surgery, the MPTP-saline group did not exhibit the same general hypoactivity on the open field test as evidenced $24 \mathrm{~h}$

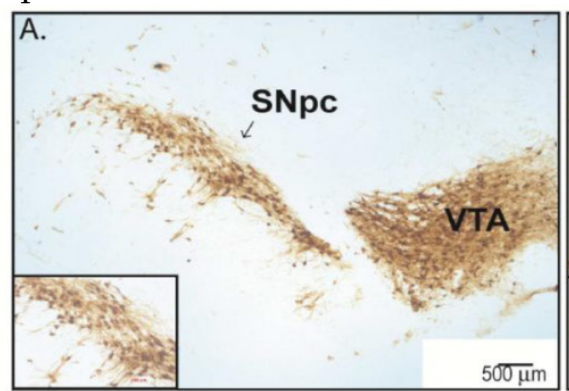

Immediately after lesion
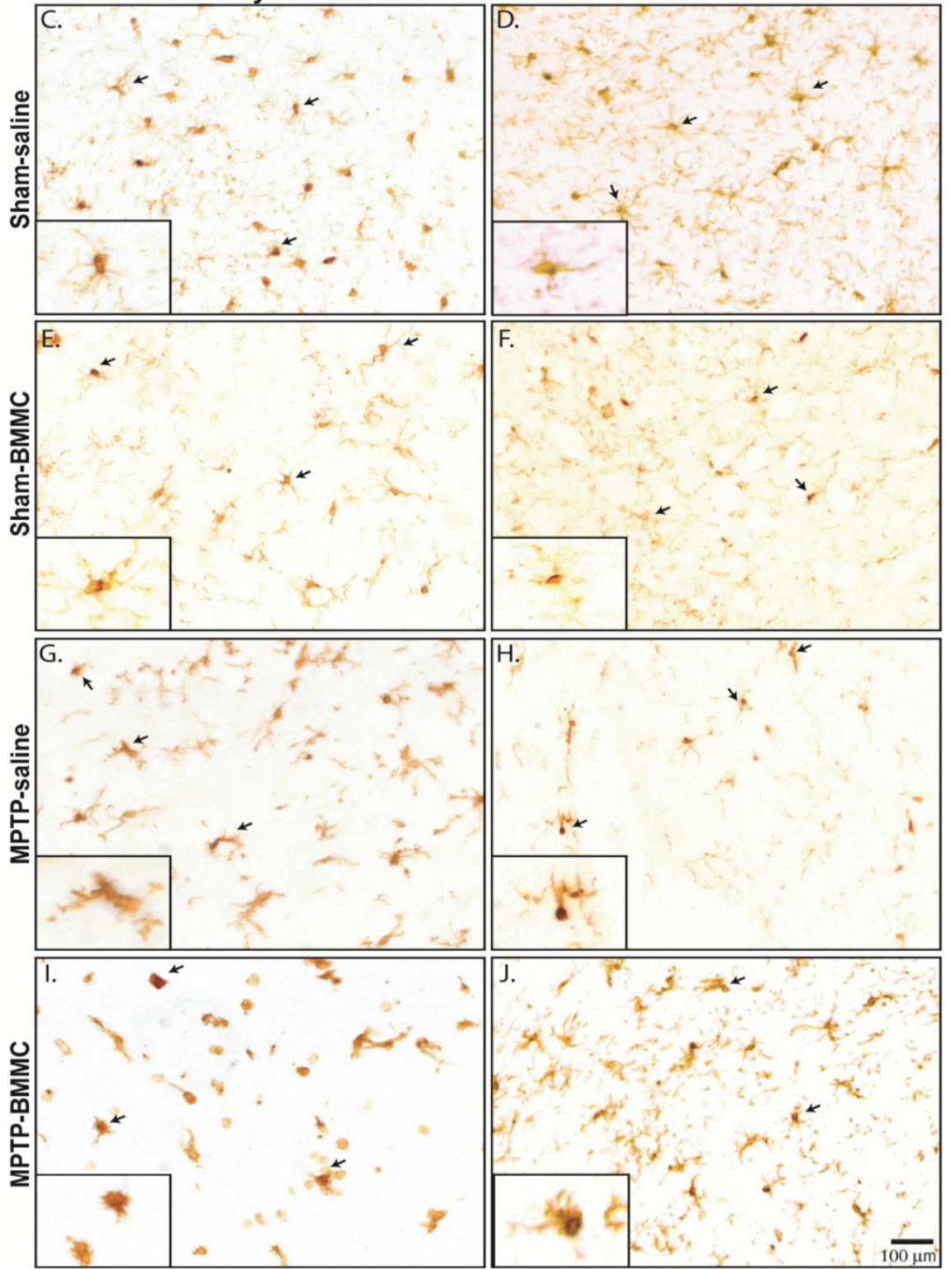

Figure 7. Photomicrography of representative sections demonstrating the different morphological patterns of microglial cells in the ventral tegmental area (VTA) (Panel 7B) and substantia nigra pars compacta (SNpc) $24 \mathrm{~h}$ (Panel 7C-I) and 8 days (Panel 7D-J) after stereotaxic surgery. Arrows indicate Ibal+ microglial cells. Scale bar: 500 um. N=3 animals/group. A) Photomicrography of a tyrosine-hydroxylase-immunostained section illustrating the investigated area. Scale bar: $100 \mu \mathrm{m}$. Note on the insets the many fine processes and the small cell body of resting non-activated lbal I microglial cells in Sham non-injured animals (7C-F). On the other hand, note the reactive state of microglial cells characterized by a bushy, hyper-ramified or amoeboid morphology (Figure 7G-J). In addition, the presence of activated microglial cells was more abundant in MPTP-lesioned rats transplanted with BMMC immediately after lesion than animals lesioned with MPTP and treated with saline (Figure 7l). after neurotoxic lesion induction, and some recovery of motor activity parameters was observed in the MPTP-lesioned rats. Many studies have demonstrated that MPTP injury in rats is transient and that recovery is common in this animal model of PD [3,34].

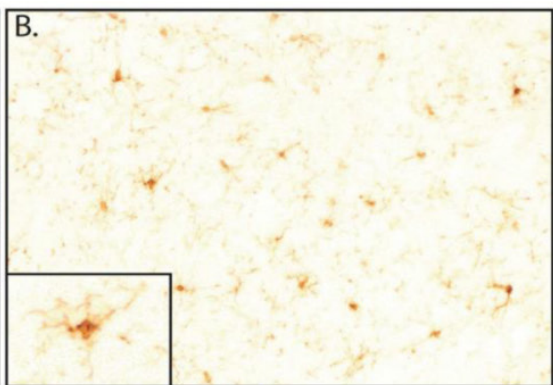

24 hours after lesion
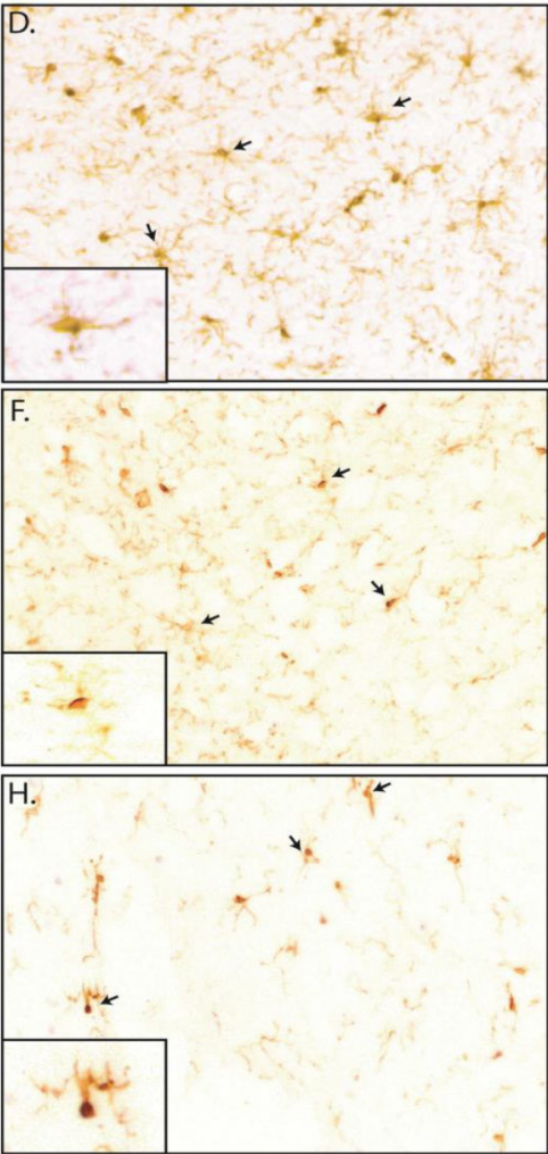

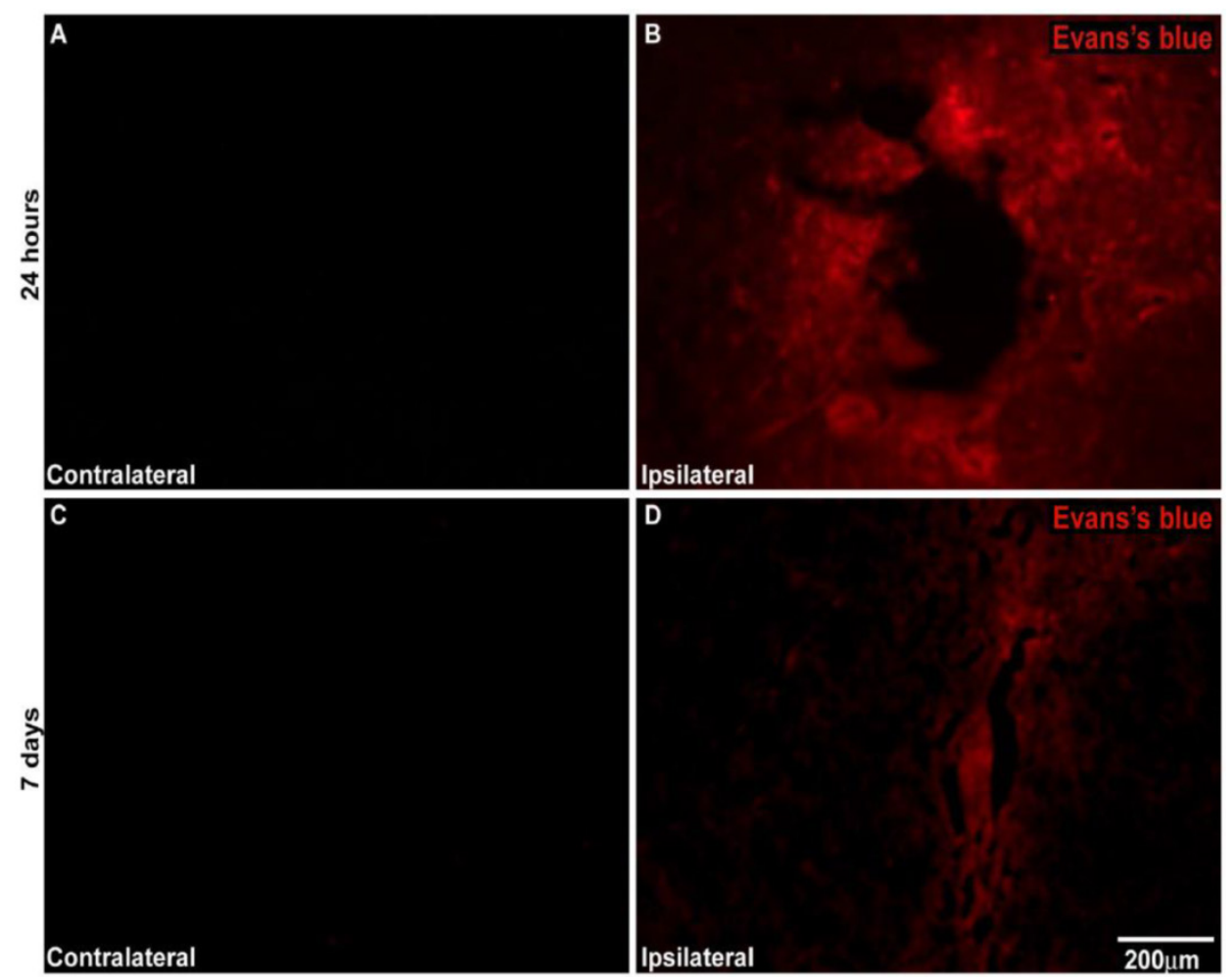

Figure 8. A) Representative photomicrography showing BBB disruption after unilateral MPTP-induced lesion creation in the SNpc of rats. $A$ and $C$ represent the contralateral side of MPTP-lesioned rats injected with Evans blue at $24 \mathrm{~h}$ and 7 days after surgery, respectively. $B$ and $D$ represent the ipsilateral side of MPTP-lesioned rats injected with Evans blue $24 \mathrm{~h}$ and 7 days after surgery, respectively. $\mathrm{N}=2$ animals for experiment. Scale bar: $200 \mu \mathrm{m}$.

Evidence suggests that nigral dopaminergic neurons are highly vulnerable to oxidative damage [40]. In fact, many clinical and animals studies, including studies involving MPTP, have reported the involvement of microglial activation, increased inflammatory mediators and ROS in the pathology of PD [40-43]. Moreover, several studies have reported that the generation of ROS and neuroinflammation can increase BBB permeability and can support, via the increased expression of inflammatory cytokines and chemokines, the entry of immune cells/blood cells, such as monocytes, lymphocytes and B cells, into the central nervous system (CNS) compartment [44-46]. Although the role played by CNS-infiltrating monocytes in neurodegenerative disease remains unclear, bone marrow-derived cells appear to be significantly recruited to regions with CNS degeneration; in some cases, this infiltration can contribute to neurodegeneration [46].

Our results investigating BBB breakdown using EB demonstrated that MPTP administration resulted in an increase of the BBB permeability both $24 \mathrm{~h}$ and 7 days after MPTP infusion, as indicated by the elevated extravasation of EB dye in the brain parenchyma surrounding the injury site. There was no extravasation of EB at the contralateral site despite of the saline injection in any of the animals investigated. Data from the literature suggest that patients with PD present with compromised BBB integrity and that MPTP administration in animal models has resulted in a significant leakage of the BBB $[9,47]$. Our observation of a significant elevation of $\mathrm{CD} 45^{+}$cells in the injury site in MPTP-BMMC animals appeared to reinforce the role played by the BBB breakdown.

Regarding transplantation therapy, our results demonstrated that animals transplanted with BMMCs and BM-MSCs immediately after MPTP injection did not exhibit significant differences in general motor activity (open field test) compared to the MPTP-saline animals. However, a histological analysis of the MPTP-BMMC animals demonstrated a pronounced loss of TH-ir cells $24 \mathrm{~h}$ after stereotaxic surgery compared to the MPTP-saline group.

We speculate that BBB impairment associated with BMMC transplantation could induce the inflammation into the brain, perhaps through microglial activation and/or peripheral inflammatory factors.

Franco and coworkers investigated the effect of BMMC transplantation and microglial modulation after stroke and suggested that an exacerbated activation of microglial cells impaired the beneficial effects of BMMC transplantation in an ischemic model [48].

Microglial cells are a type of glial cell derived from myeloid precursors of bone marrow during the development; these cells play an important role in the CNS by detecting physiological disturbances through 
numerous molecular receptors. Recent studies have demonstrated that the expression of cellular adhesion molecules was up-regulated by microglial activation; moreover, the subsequent induction of chemokines resulted in peripheral leucocytes chemoattracted to the site of injury $[45,49]$. In addition, other studies have demonstrated that before injury, microglial cells can become over-activated or primed, which can result in an exaggerated pro-inflammatory response in the presence of additional stimuli [50,51].

Dopaminergic neurons in the SNpc are particularly susceptible to microglia-induced neurotoxicity due to the high density of microglial cells in this brain site $[52,53]$ and a study carried by Ouchi and coworkers revealed that microglial activation occurred in patients with early PD [54].

Our histological data also showed that 8 days after stereotaxic surgery, the treatment with BMMC $24 \mathrm{~h}$ after MPTP injury and MPTP-lesioned rats treated with saline exhibited a similar loss of $\mathrm{TH}$-immunoreactivity in the SNpc. These data suggest that BMMC transplantation immediately after MPTP injury induced an acceleration of the lesion but did not have any effect on the final number of TH-ir cells when BMMCs were transplanted when the injury was already present (24 h after MPTP infusion).

MPTP neurotoxin causes initial disturbance in function of dopaminergic neuron due to mitochondrial dysfunctional and oxidative species production [1]. In response to initial perturbation and once that microglial cells are involved in MPTP metabolism, multiple glial pathway are activated resulting in glutamate release, more oxidative species, growth factors, pro-inflammatory cytokines, including interleukine 6 (IL-6), IL-1, and tumor necrosis factor a (TNF-a), upregulation of inducible nitric oxide synthase (iNOS) and enzyme cyclooxygenase type 2 (COX-2) $[55,56,43]$. Lima and coworkers demonstrated a significant increase in the COX-2 protein expression $16 \mathrm{~h}$ after MPTP infusion at SNpc and that after this time-point, levels of COX-2 decreased progressively revealed no difference $24 \mathrm{~h}, 3$ and 7 days after MPTP infusion in comparison to control group [43]. This finding supports our suggestion that BMMCs transplanted immediately to MPTP infusion can be recruited by injury site, pump cytokines that might act directly or indirectly through the stimulation of the resident glial cells and acutely accelerate neuronal injury. In fact, our histological data illustrate the change of microglial morphology from their resting state to a reactive amoeboid state (activated microglia) in the SNpc of the animals lesioned with MPTP transplanted immediately with BMMC. McGeer and coworkers described the extensive presence of reactive amoeboid microglia in postmortem study of PD indicating that these cells could lead to neurodegeneration of SN [57].

In the present study, CM-DiI-labeled BMMCs were detected in brain slices from transplanted animals at all investigated intervals. Moreover, CD45-positive cells, which can identify nucleated white blood cells that have recently infiltrated the brain, were also observed in brain slices from all animals, though an elevation of CD45-positive cells has been reported for MPTP-lesioned animals transplanted with BMMCs [58]. Thus, the loss of TH-ir cells in the $\mathrm{SNpc}$ associated with microglial activation might be exacerbated by the infiltration of transplanted BMMCs and/or systemic inflammatory molecules due to increased BBB permeability.

In line with our explanation, a study by Kokovay and Cunningham demonstrated that endogenous cells derived from bone marrow could gain access to the adult brain. Specifically, these authors demonstrated that endogenous bone marrow-derived cells in MPTP-lesioned animals could infiltrate into the brain and express the microglia marker C11b [59].

In addition, our results showed for the first time that systemic BM-MSC transplantation in MPTP-lesioned rats resulted in protection against dopaminergic neuronal damage, even though this apparent protection did not result in an improvement of general motor alterations.

Studies have described BM-MSC transplantation as a promising approach for the treatment of neurological disorders due to the tissue-repair potential and "homing" capability of these cells to the injured area (see reference 60 for further reviewing). At the injury site, these cells can release many trophic factors that accelerate the endogenous repair and control the local inflammation associated with neuronal degeneration [60]. In fact, several studies have investigated the effect of mesenchymal stem cells or bone marrow stromal cells in neurodegenerative diseases $[9,20,61,62]$. Particularly for PD, many studies have reported the beneficial effects of BM-MSC transplantation in PD animal models; these animals showed behavioral recovery after the transplantation and protection of the dopaminergic neurons $[9,61]$. Thus, the protective effect of MSC transplantation on the number of TH-ir cells after MPTP injury may be related to the immunomodulatory and anti-inflammatory properties previously described in literature $[10,25]$. Our results reinforce these beneficial effects of BM-MSCs, even when these cells were systemically endovenously administered.

Our study presents a major limitation. The MPTP injury does not reproduce exactly the PD and several limitations are presented in this model of PD, including absence eosinophilic inclusions resembling 
Lewys bodies, partial lesion produced by neurotoxin and early studies reporting that rodents are more resistant to MPTP [63]. In fact, MPTP-lesioned rats showed time course changes in motor activity such as initial hypokinesia and later recovery of behavioral alterations as a consequence of partial lesion and development to compensatory mechanism in D1 dopamine receptors [64]. Despite of that, however, it has been considered a powerful tool to understanding of early events causing the nigral degeneration of PD [63]. In addition, since the 24 hours or seven days' time frames after MPTP injection used in our study were too short, another major limitation of the present study was our inability to predict the real neuronal loss in a longer period of observation, since the acute loss of TH-immunoreactivity could represent more a neuronal damage due to the inflammatory process, reactive microglia, up-regulation of a broad array of inflammatory cytokines and ROS $[56,63]$ rather than a real dopaminergic neuronal loss.

In summary, our study showed that systemic BMMC transplantation in MPTP-lesioned rats immediately after neurotoxin infusion induced an additional loss of TH-immunoreactivity and a worsening of associated depressive-like behavior. These findings were associated with an increased presence of $\mathrm{CD} 45^{+}$ cells and activated Iba1+ microglial cells in the injury site and breakdown of the BBB. In contrast, systemic BM-MSC transplantation showed promising effects in the protection against dopaminergic neuron injury. Taken together, our data suggest that we must be cautious when considering cell therapy with BMMCs instead of BM-MSCs to treat and/or prevent PD. Moreover, additional studies are necessary to elucidate the cellular and molecular mechanisms of this action.

\section{Abbreviations}

BBB: blood brain barrier; BMMC: bone marrow mononuclear cells; BM-MSC: bone marrow derived mesenchymal stem cell; MPTP: 1-methyl-4-phenyl1,2,3,6-tetrahydropyridine; PD: Parkinson's disease; ROS: reactive oxygen species; $\mathrm{TH}$ : tyrosine hydroxylase.

\section{Acknowledgements}

This work was supported by CAPES, CNPq, FUNEPU and AFIP (Brazil).

\section{Author contributions}

All authors participated in the design and interpretation of the studies, analysis of the data and review of the manuscript. CSC, CSL, LFO and ACA conducted the experiments; JB conducted the $\mathrm{TH}$ immunohistochemistry analysis; and CSC, MABFV and VJD wrote the manuscript.

\section{Conflict of Interest}

The authors have declared that no conflict of interest exists.

\section{References}

1. Dauer W, Przedborski S. Parkinson's disease: mechanism and models. Neuron. 2003;39:889-909.

2. Chaudhurt KR, Schapira AH. Non-motor symptoms of Parkinson's disease: dopaminergic pathophysiology and treatment. Lancet Neurol. 2009;8:464-474.

3. Grinberg LT, Rueb U, Alho AT, Heinsen H. Brainstem pathology and non-motor symptoms in PD. J Neurol Sci. 2010;289:81-88.

4. Ferrer I. Early involvement of the cerebral cortex in Parkinson's disease: convergence of multiple metabolic defects. Prog Neurobiol. 2009;88:89-103.

5. Da Cunha C, Gevaerd MS, Vital MA, Miyoshi E, Andreatini R, Silveira R, Takahashi RN, Canteras NS. Memory disruption in rats with nigral lesions induced by MPTP: a model for early Parkinson's disease amnesia. Behav Brain Res. 2001;124:9-18.

6. Capitelli C, Sereniki A, Lima MM, Reksidler AB, Tufik S, Vital MABF. Melatonin attenuates tyrosine hydroxylase loss and hypolocomotion in MPTP-lesioned rats. Eur J Pharmacol. 2008;594:101-108.

7. Gao HM, Liu B, Zhang W, Hong JS. Novel anti-inflammatory therapy for Parkinson's disease. Trend Pharmacol Sci. 2003;24:395-401.

8. Takahashi H, Wakabayashi K. Controversy: Is Parkinson's disease entity? Yes. Parkinsonism Relat Disord. 2005;11:S31-S37.

9. Schulz JB, Falkenburger BH. Neuronal pathology in Parkinson's disease. Cell Tissue Res. 2004;318:135-147.

10. Chao YX, He BP, Tay SS. Mesenchymal stem cell transplantation attenuates blood brain barrier damage and neuroinflammation and protects dopaminergic neurons against MPTP toxicity in the substantia nigra in a model of Parkinson's disease. J Neuroimmunol. 2009;216:39-50.

11. Lloyd KG, Davidson L, Hornykiewicz O. The neurochemistry of Parkinson's disease: effect of L-dopa therapy. J Pharmacol Exp Ther. 1975;195:453-464.

12. Poewe W, Mahlknecht P. The Clinical progression of PD. Parkinsonism Relat Disord. 2009;15:S28-32.

13. Piccini P, Brooks DJ, Björklund A, Gunn RN, Grasby PM, Rimoldi O, Brundin $\mathrm{P}$, Hagell $\mathrm{P}$, Rehncrona S, Widner $\mathrm{H}$, Lindvall O. Dopamine release from nigral transplants visualized in vivo a Parkinson's patient. Nat Neurosci. 1999;2:1137-1140.

14. Lu D, Mahmood A, Wang L, Li Y, Lu M, Chopp M. Adult bone marrow stromal cells administered intravenously to rats after traumatic brain injury migrate into brain and improve neurological outocome. Neuroreport. 2001; 12:559-63.

15. Giraldi-Guimarães A, Rezende-Lima M, Bruno FP, Mendez-Otero R. Treatment with bone marrow mononuclear cells induces functional recovery and decreases neurodegeneration after sensorimotor cortical ischemia in rats. Brain Res. 2009;1266:108-120.

16. Shintani A, Nakao N, Kakishita K, Itakura T. Protection of dopamine neurons by bone marrow stromal cells. Brain Res. 2007;1186:48-55.

17. Wijeyekoon R, Barker RA. Cell replacement therapy for Parkinson's disease. Biochim Biophys Act. 2009;1792:688-702.

18. Lindvall $\mathrm{O}$, Brundin $\mathrm{P}$, Widner H, Rehncrona S, Gustavii B, Frackowiak R, Leenders KL, Sawle G, Rothwell JC, Marsden CD, et al. Grafts of fetal dopamine neurons survive and improve motor function in Parkinson's disease. Science. 1990;247:574-7.

19. Kim JH, Auerbach JM, Rodríguez-Gómez JA, Velasco I, Gavin D, Lumelsky N, Lee SH, Nguyen J, Sánchez-Pernaute R, Bankiewicz K, McKay R. Dopamine neurons derived from embryonic stem cells function in an animal model of Parkinson's disease. Nature. 2002;418:50-6.

20. Danielyan L, Schäfer R, Von Ameln-Mayerhofer A, Bernhard F, Verleysdonk S, Buadze M, Lourhmati A, Klopfer T, Schaumann F, Schmid B, Koehle C, Proksch B, Weissert R, Reichardt HM, van den Brandt J, Buniatian GH, Schwab M, Gleiter $\mathrm{CH}$, Frey WH. Therapeutic efficacy of intranasally delivered mesenchymal stem cells in a rat model of PD. Rejuvenation Res. 2011;14:1-14.

21. Sayles M, Jain M, Barker RA. The cellular repair of the brain in Parkinson's disease - past, present and future. Transpl Immunol. 2004:12:321-42.

22. Hall VJ, Li JY, Brundin P. Restorative cell therapy for PD: a quest for the perfect cell. Semin Cell Dev Biol. 2007;18:859-869.

23. Orkin SH. Diversification of hematopoietic stem cells to specific lineages. Nat Rev Genet. 2000;1:57-64.

24. Weissman IL, Anderson DJ, Gage F. Stem and progenitor cells: origins, phenotypes, lineage commitments, and transdifferentiations. Annu Rev Cell Dev Biol. 2001;17:387-403.

25. Singer NG and Caplan AI. Mesenchymal stem cells: Mechanism of inflammation. Annu Rev Pathol Mech Dis. 2011;6:457-78.

26. Strauer BE and Ran KS. Stem cell therapy in perspective. Circulation. 2003; 107:929-934. 
27. Shetty P, Ravindran G, Sarang S, Thakur AM, Rao HS, Viswanathan C. Clinical grade mesenchymal stem cells transdifferentiated under xenofree conditions alleviates motor deficiencies in a rat model of Parkinson's disease. Cell Biol Int. 2009;33:830-8

28. Venkataramana, NK, Kumar SK, Balaraju S, Radhakrishnan RC, Bansal A, Dixit A, Rao DK, Das M, Jan M, Gupta PK, Totey SM. Open-labeled study of unilateral autologous bone-marrow-derived mesenchymal stem cell transplantation in Parkinson's disease. Transl Res. 2010;155:62-70.

29. Thomas MG, Stone L, Evill L, Ong S, Ziman M, Livia H. Bone marrow stromal cells as replacement for Parkinson's disease: generation of an anatomical but not functional neuronal phenotype. Transl Res. 2011;157:56-63.

30. Lindvall O, Björklund A. Cell Therapy in Parkinson's disease. NeuroRx. 2004;1:382-93.

31. Paxinos G, Watson C. The rat brain in stereotaxic coordinates. San Diego: Academic Press. 1986

32. Neuhuber B, Swanger SA, Howard L, Mackay A, FISCHER I. Effect of platting density and culture time on bone marrow stromal cell characteristic. Exp Hematol. 2008;36:1176-1185.

33. Broadhurst PL. Experiments in psychogenetics. In: Eisenk H.J, Ed. Experiments in personality. London: Routledge and Kegan Paul. 1960: 52-71.

34. Sedelis M, Schwarting RKW, Huston JP. Behavioral phenotyping of the MPTP mouse model of Parkinson's disease. Behav Brain Res. 2001;125:109-125.

35. Lucki I. The forced swimming test as a model for core and component behavoral effects of antidepressant drugs. Behav Pharmacol. 1997;8:523-532

36. Kim Y, Park H, Lee G, Bang O, Ahn Y, Joe E, Kim H, Lee P. Neuroprotective effects of human mesenchymal stem cells on dopaminergic neurons through anti-inflammatory action. Glia. 2009;57:13-23.

37. Wirenfeldt M, Clare R, Tung S, Bottini A, Mathern G, Vinters H. Increased activation of Iba1 ${ }^{+}$microglia in pediatric epilepsy patients with Rasmussen's encephalitis compared with cortical dysplasia and tuberous sclerosis complex. Neurobiol Dis. 2009;34:432-440.

38. Strbian D, Durukan A, Pitkonen M, Marinkovic I, Tatlisumak E, Pedrono E, Abo-Ramadan U, Tatlisumak T. The blood-brain barrier is continuously open for several weeks following transient focal cerebral ischemia. Neurosci. 2008; 153(1):175-81.

39. Heikkila RE, Hess A, Duvoisin RC. Dopaminergic neurotoxicity of 1-methyl-4-phenyl-1,2,3,6-tetrahydropyridine in mice. Science. 1984;224:1451-1453.

40. Collins LM, Toulouse A, Connor TJ, Nolan YM.. Contributions of central and systemic inflammation to the pathophysiology of PD. Neuropharmacol. 2012;62:2154-2168.

41. Imamura K, Hishikawa N, Sawada M, Nagatsu T, Yoshida M, Hashizume Y. Distribution of major histocompatibility complex class II-positive microglia and cytokine profile of Parkinson's disease brains. Acta Neuropathol. 2003:106:518-526.

42. McGeer PL, Schwab C, Parent A, Doudet D. Presence of reactive microglia in monkey substantia nigra years after MPTP administration. Ann Neurol. 2003;54:599-604.

43. Lima MMS, Reksidler AB, Zanata SM, Machado HB, Tufik S, Vital MABF. Different parkinsonism models produced time-dependent induction COX-2 in the substantia nigra of rats. Brain Res. 2006;1101:117-125.

44. Hathaway CA, Percy WH, Williams JL. Effects of free radicals and leukocytes on increases in blood-brain barrier permeability during colitis. Dig Dis Sci. 2000;45:967-75.

45. Chung YC, Ko HW, Bok E, Park ES, Huh SH, Nam JH, Jin BK. The role of neuroinflammation on the pathogenesis of PD. BMB Rep. 2010;43:225-232.

46. Brochard V, Combadière B, Prigent A, Laouar Y, Perrin A, Beray-Berthat V, Bonduelle O, Alvarez-Fischer D, Callebert I, Launay JM, Duyckaerts C, Flavell RA, Hirsch EC, Hunot S. Infiltration of $\mathrm{CD}^{+}$lymphocytes into the brain contributes to neurodegeneration in a mouse model of Parkinson's disease. J Clin Invest. 2009;119:182-192.

47. Kortekaas R, Leenders KL, Van Oostrom JC, Vaalburg W, Bart J, Willemsen AT, Hendrikse NH. Blood brain barrier dysfunction in Parkinsonian midbrain in vivo. Ann Neurol. 2005;57:176-179.

48. Franco EC, Cardoso MM, Gouvêia A, Pereira A, Gomes-Leal W. Modulation of microglial activation enhances neuroprotection and functional recovery derived from bone marrow mononuclear cell transplantation after cortical ischemia. Neurosci Res. 2012;73:122-132.

49. Stone DK, Reynolds AD, Mosley RL, Gendelman HE. Innate and adaptive immunity for the pathobiology of Parkinson's disease. Antioxid Redox Signal. 2009;11:2151-2166.

50. McGeer EG, Klegeris A, McGeer PL. Inflammation, the complement system and diseases of aging. Neurobiol Aging. 2005;26:94-97.

51. Doorn KJ, Lucassen PJ, Boddeke HW, Prins M, Berendse HW, Drukarch B, van Dam AM. Emerging roles of microglial activation and non-motor symptoms in Parkinson's disease. Prog in Neurobiol. 2012;98:222-238.

52. Lawson LJ, Perry VH, Dri P, Gordon S. Heterogeneity in the distribution and morphology of microglia in the normal adult mouse brain. Neurosc. 1990:39:151-170.

53. Kim WG, Mohney RP, Wilson B, Jeohn GH, Liu B, Hong JS. Regional difference in susceptibility to lipopolysaccharide-induced neurotoxicity in the rat brain: role of microglia. J Neurosc. 2000;20:6309-6316.
54. Ouchi $Y$, Yoshikawa E, Sekine $Y$, Futatsubashi M, Kanno T, Oqusu T, Torizuka T. Microglial activation and dopamine terminal loss in early Parkinson's disease. Ann Neurol. 2005;57:168-75.

55. Liberatore GT, Jackson-Lewis V, Vukosavic S, Mandir AS, Vila M, McAuliffe WG, Dawson VL, Dawson TM, Przedborski S. Inducible nitric oxide synthase stimulates dopaminergic neurodegeneration in the MPTP model of PD. Nat Med. 1999;5:1403-09.

56. McGeer PL, Yasojima K, McGeer EG. Inflammation in PD. Adv Neurol. 2001;86:83-9.

57. McGeer PL, Itagaki S, Boyes BE, McGeer EG. Reactive microglia are positive for HLA-DR in the substantia nigra of Parkinson's and Alzheimer's disease brains. Neurology. 1988;38:1285-91.

58. Cosenza-Nashat MA, Kim MO, Zhao ML, Suh HS, Lee SC. CD45 isoform expression in microglia and inflammatory cells in HIV-1 encephalitis. Brain Pathol. 2006;16:256-265.

59. Kokovay E, Cunningham A. Bone marrow-derived microglia contribute to the neuroinflammatory response and express iNOS in the MPTP mouse model of Parkinson's disease. Neurobiol. 2005;19:471-478.

60. Glavaski-Joksimovic A, Bohn MC. Mesenchymal stem cells and neuroregeneration in Parkinson's disease. Exp Neurol. 2013;247:25-38

61. Hellman MA, Panet H, Barhum Y, Melamed E, Offen D. Increased survival and migration of mesenchymal bone marrow stem cells in 6-hydroxydopamine-lesioned rodents. Neurosci Lett. 2006;395:124-128.

62 Lee JK, Jin HK, Endo S, Schuchman EH, Carter JE, Bae JS. Intracerebral transplantation of bone marrow-derived mesenchymal stem cells reduces amyloid-beta deposition and rescues memory deficits in Alzheimer's disease mice by modulation of immune responses. Stem cell. 2010;28:329-343.

63. Speciale SG. MPTP Insights into parkinsonian neurodegeneration. Neurotoxicol Teratol. 2002;24:607-620.

64. Perry JC, Hipólide DC, Tufik S, Martins RD, Da Cunha C, Andreatini R, Vital MA. Intra-nigral MPTP lesion in rats: Behavioural and autoradiography studies. Exp Neurol. 2005;195:322-329. 